\title{
TOWARDS ENERGY EFFICIENT LATENT HEAT STORAGE TANKS WITH HEAT TRANSFER FLUID CHANNELS AND FINITE INLET POWER
}

\author{
Bart Peremans, ${ }^{1,2, *}$ Maarten Blommaert, ${ }^{1,2}$ Jan Diriken, ${ }^{2,3}$ Martine Baelmans ${ }^{1,2}$ \\ ${ }^{1}$ KU Leuven, Department of Mechanical Engineering, Leuven, 3001, Belgium \\ ${ }^{2}$ EnergyVille, Genk, 3600, Belgium \\ ${ }^{3}$ Flemish Institute for Technological Research (VITO), Mol, 2400, Belgium
}

\begin{abstract}
In this research, latent heat storage tanks are modelled with a water flow with a finite total heat capacity. Because of the finite heat capacity of the water flow, the Phase Change Material (PCM) will not melt uniformly. This is in contrast to the case were large water flows induce a constant wall temperature for the PCM. In this paper, we show that increased charging efficiencies can be obtained for high flow lengths and reduced PCM layer thicknesses. Additionally, both storage and charging performance increase for lower water volume fractions. Further, we show that multiple stage design enables improved charging performance and reduced pumping power requirements.
\end{abstract}

KEY WORDS: Latent heat storage, Phase Change Material, charging efficiency

\section{INTRODUCTION}

Renewable energy systems are able to reduce the classical heating units, which burn fossil fuels and emit hazardous greenhouse gasses [1]. Unfortunately, these renewables are most often strongly intermittent and as such might not be available at the time of demand need. Balancing supply and demand of thermal energy therefore becomes a challenge. Introducing compact and efficient thermal energy storages is a way to tackle this problem [2]. Most of the thermal energy stored today, relies on the principle of sensible heat. Therefore, temperature changes are needed to store and release heat. To store large amounts of heat, either large temperature changes or large storage volumes are needed. In contrast, latent heat storages use Phase Change Materials (PCMs) which are allowed to melt and solidify. This allows to reach high storage capacities within a small temperature range [3]. The commonly known drawback of PCMs, however, is their low thermal conductivity, which hampers the charging and discharging performance of latent heat storages. To overcome this problem, recent studies have focused on increasing the charging performance of latent heat storages by means of PCM encapsulation or by introducing highly conductive fin structures [4]. The former is based upon reducing the diffusive length in the PCM, i.e. the maximum distance between PCM and a heat exchanging surface, while the latter's target is to increase the overall thermal conductivity. Most of these studies are performed using a constant wall temperature heat supply $[4,5]$ or using an imposed wall heat flux [6], because of the ease of implementation. In the first case, as the wall is at a constant temperature, it serves as a heat source with an infinite heat capacity. In the second case, an imposed constant wall heat flux leads to a finite heat source, without any restriction on wall temperatures. However, in realistic heat storages, a heat transfer fluid, such as water, with a finite total heat capacity acts as the heat source. The limited total heat capacity introduces temperature gradients in the flow

*Corresponding Bart Peremans: bart.peremans@kuleuven.be 
and thus also influences the wall temperature.

In this paper, the performance of latent heat storages charged by a heat transfer fluid with a finite total heat capacity is investigated, based on numerical studies and dimensional analysis. Moreover, based upon these results, latent heat storages are designed with high storage and charging performance by changing the shape of the storage and its PCM modules only. The first section introduces the performance indicators that are used to define both the storage and charging performance of the storage. In the second section, the model equations describing the melting of the PCM and the heat supply by the water are discussed. The enthalpy method is used to deal with the phase change of the PCM, whereas a reduced energy model is set up for the heat transfer in the water flow. The third section includes a dimensional analysis, which is carried out to include the dependencies of the water flow properties on the storage's performance. In this way, a general framework for the charging performance of latent heat storages is created in dimensionless format. In the fourth section, a parameter study reveals the charging efficiency trends for different PCM domain aspect ratios, heat inputs as well as water channel widths. Moreover, the results are afterwards translated to performance trends for multiple stage designs. The last section provides a summary of the results, conclusions, and future work.

\section{STORAGE PERFORMANCE}

The performance of latent heat storages is expressed by two main quantities [4]. On the one hand, the storage capacity, defined as the storage or energy density, is a measure of the amount of heat that can be stored in a certain storage volume. Storage density is based on the latent heat storage capacity and is therefore expressed as

$$
e=\frac{E}{V_{t o t}}=\rho \Delta h \frac{V_{\mathrm{PCM}}}{V_{t o t}},
$$

with $\rho$ the PCM density, $\Delta h$ the PCM latent heat and $V_{\mathrm{PCM}}$ and $V_{\text {tot }}$ the PCM volume and total storage volume respectively. The high latent heat of fusion of PCMs enables high storage densities to be reached.

On the other hand, the power density is defined as the mean power per unit storage volume at which the storage is being charged or discharged, i.e.

$$
\dot{q}=\frac{\dot{Q}}{V_{t o t}}=\frac{e}{t_{c h}},
$$

with $\dot{Q}$ the mean charging power and $t_{c h}$ the charge time, i.e. the total time needed to melt or solidify all the PCM in the storage. High power densities are achieved by reducing the PCM characteristic length and by inserting highly conductive fin structures [4]. Another way of representing the charging performance of the storage is by means of a charging efficiency. Charging efficiency is the ratio of stored heat to input heat:

$$
\eta=\frac{E}{E_{\text {in }}}=\frac{\dot{q} \cdot V_{t o t}}{\dot{Q}_{\text {in }}}
$$

with $\dot{Q}_{i n}=\dot{m} c_{w}\left(T_{i n}-T_{m}\right)$ the available input power supplied by the heat transfer fluid and $E_{i n}=\dot{Q}_{i n} \cdot t_{c h}$ the accumulated energy input provided by the heat transfer fluid at the storage inlet. Here, $\dot{m}, c_{w}$ and $T_{i n}$ are defined as the mass flow rate, specific heat capacity and input temperature of the heat transfer fluid respectively, while $T_{m}$ stands for the melting temperature of the PCM. Charging efficiency determines the fraction of heat that could effectively be stored as latent heat and therefore always ranges between zero and one. This performance indicator is later employed in section 5.

\section{MODELLING EQUATIONS}

The complete simulation domain is composed of a rectangular PCM domain and a parallel plate water channel. The water channel is positioned below the PCM and water is flowing from left to right in all cases (see figure 


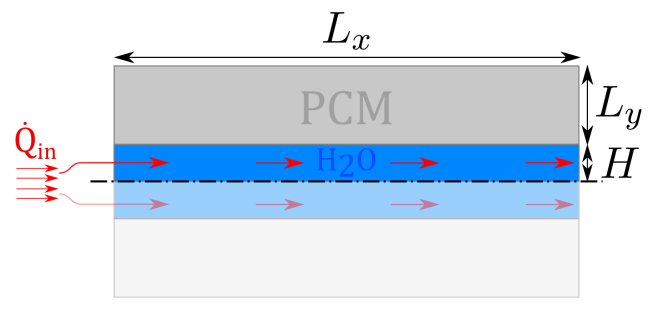

(a) Single stage.

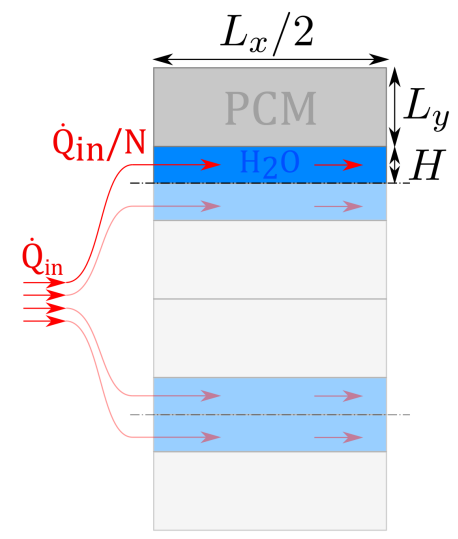

(b) Multiple stage.

Fig. 1 Single stage and multiple stage designs with the red arrows indicating the water flow.

1(a)). In reality, the water channel is always placed in the middle of the storage tank with PCM surrounding it. Therefore, only half of the water channel is simulated. The dashed line in figure 1(a) acts as a symmetry plane. All the PCM boundaries are assumed to be perfectly insulated, except for the interface with the water channel where heat transfer takes place. A constant mass flux flows through the channel with a constant input temperature, therefore fixing the input power $\left(\dot{Q}_{i n}\right)$.

Different model equations are used for the PCM region and the water channel flow. For both sets of model equations, the finite volume technique is applied. The discretized system of equations is solved for the unknown temperatures and enthalpies. Time-marching enables all transient behaviour to be captured using the implicit Euler method. All simulations are performed in two dimensions on a uniform rectangular grid.

In this section, the model equations for solving the heat transfer and melting/solidification process in PCMs are shown first. Secondly, a reduced energy model is presented for efficiently solving the heat supply by the water flow.

\subsection{PCM state change}

The main heat transfer phenomenon taking place in PCMs is heat conduction. Therefore the transient heat conduction equation is solved, i.e.

$$
\rho_{\mathrm{PCM}} \frac{\partial h}{\partial t}-\nabla \cdot k_{\mathrm{PCM}} \nabla T=0 .
$$

Here $h, T, \rho_{\mathrm{PCM}}$ and $k_{\mathrm{PCM}}$ are the PCM enthalpy, temperature, density, and thermal conductivity respectively.

To capture the phase change of the PCM, the enthalpy method [7] is applied, which couples the PCM temperature and enthalpy according to its phase:

$$
h=\left\{\begin{array}{ll}
c_{p, \mathrm{PCM}} T & : T<T_{m} \\
c_{p, \mathrm{PCM}} T+\Delta h & : T>T_{m}
\end{array},\right.
$$

with $c_{p, \mathrm{PCM}}$ being the heat capacity, $\Delta h$ the latent heat of fusion and $T_{m}$ the melting temperature of the PCM.

\subsection{Water flow}

To model the heat transfer in the water flow, a transient convection-diffusion equation is solved. To limit the computational cost, two assumptions are made. On the one hand, instead of solving a complex two-dimensional transient flow field, the water flow is assumed to be hydrodynamically fully developed. For a flow between 
infinite parallel plates, the velocity profile is therefore known [8]. On the other hand, modelling the transient temperature distribution in the water flow requires a fine grid to get an acceptable resolution. This is associated with a high computational cost and long simulation times. To reduce the computational cost, a reduced 1D energy model is derived by height-averaging the energy equation [9]:

$\frac{\partial \rho_{w} c_{w}\langle T\rangle}{\partial t}+\langle\zeta \xi\rangle \frac{\partial}{\partial x}\left[\rho_{w} c_{w}\langle v\rangle\langle T\rangle\right]+(1-\langle\zeta \xi\rangle) \frac{\partial}{\partial x}\left[\rho_{w} c_{w}\langle v\rangle T_{i}\right]-\frac{\partial}{\partial x}\left[k_{w} \frac{\partial\langle T\rangle}{\partial x}\right]-k_{w}\left(\langle T\rangle-T_{i}\right)\left\langle\frac{\partial^{2} \xi}{\partial y^{2}}\right\rangle=0$,

with $\zeta(y)$ and $\xi(y)$ being the dimensionless velocity and temperature profile respectively and $\langle$.$\rangle the averaging$ operator. Here, $T, \rho_{w}, c_{w}$ and $k_{w}$ are the water temperature, density, heat capacity and thermal conductivity respectively, $v$ the water velocity and $T_{i}$ the interface temperature.

\section{DIMENSIONAL ANALYSIS}

The dimensional analysis retrieved in [4] is extended to include the heat supply by the water flow. By showing the results in non-dimensional properties, their dependencies are generalized. As a result of the representation in non-dimensional properties, the dimensional analysis carried out enables results to be scaled to any storage size.

The water flow introduces the following extra parameters: water properties (thermal conductivity $k_{w}$, density $\rho_{w}$ and heat capacity $c_{w}$ ), mean flow velocity $\langle v\rangle$ and flow channel dimensions. For a parallel plate flow, the channel is characterized by its length $L_{x}$ and channel width $H$ (see figure 1(a)). By constructing dimensionless groups, the following non-dimensional numbers are easily found [4]:

$$
\mathrm{AR}_{x y}=\frac{L_{x}}{L_{y}}, \quad \mathrm{AR}_{x z}=\frac{L_{x}}{L_{z}}, \quad \bar{T}=\frac{T_{m}-T_{0}}{T_{i n}-T_{0}}, \quad \text { Ste }=\frac{c_{p, \mathrm{PCM}}\left(T_{i n}-T_{0}\right)}{\Delta h}, \quad \Pi_{L}=\frac{L_{x}^{2} \Delta h}{\alpha^{2}},
$$

with $L_{y}$ and $L_{z}$ the second, respectively third dimension of the PCM domain, $T_{i n}$ the supply water temperature, $T_{0}$ the initial temperature of the domain and $\alpha=\left.\frac{k}{\rho c_{p}}\right|_{\mathrm{PCM}}$ the PCM thermal diffusivity, and additional nondimensional numbers, due to the introduction of the water flow:

$$
\bar{\rho}_{w}=\frac{\rho_{w}}{\rho_{\mathrm{PCM}}}, \quad \bar{k}_{w}=\frac{k_{w}}{k_{\mathrm{PCM}}}, \quad \bar{c}_{w}=\frac{c_{w}}{c_{p, \mathrm{PCM}}}, \quad \psi=\frac{H}{L_{y}+H}, \quad \text { and } \quad P e=\frac{\langle v\rangle H}{\alpha_{w}} .
$$

Here, $\bar{k}_{w}, \bar{\rho}_{w}$ and $\bar{c}_{w}$ are the dimensionless water properties, $\psi$ is defined as the water volume fraction, $P e$ the water Péclet number and $\alpha_{w}$ the water thermal diffusivity $\left(\alpha_{w}=\frac{k_{w}}{\rho_{w} c_{w}}\right)$. The extension of the dimensional analysis results in the following relation between non-dimensional charge time and the remaining dimensionless groups (according to the Buckingham Pi theorem [10]):

$$
\bar{t}_{c h}=f\left(\mathrm{AR}_{x y}, \mathrm{AR}_{x z}, \bar{T}, S t e, \Pi_{L}, \bar{\rho}_{w}, \bar{k}_{w}, \bar{c}_{w}, \psi, P e\right), \quad \text { with } \quad \bar{t}_{c h} \triangleq \frac{\alpha t_{c h}}{L_{y}^{2}}
$$

the dimensionless charge time. The definition of the dimensionless power density $\bar{q}$ and energy density $\bar{e}$ remains unchanged, i.e.

$$
\overline{\dot{q}} \triangleq \frac{e / e_{\max }}{\alpha t_{c h} / L_{y}^{2}}=\frac{\dot{q} \cdot L_{y}^{2}}{\alpha E_{\max }}=\frac{\bar{e}}{\bar{t}_{c h}}, \quad \bar{e} \triangleq \frac{e}{e_{\max }}=\frac{V_{\mathrm{PCM}}}{V_{t o t}}=1-\psi .
$$

\section{PARAMETER STUDY}

In this section we first study the limit case of a very narrow water channel. The dependency of the charging efficiency on the PCM-domain shape and on the input power is investigated. Next, we discuss the sensitivity of 


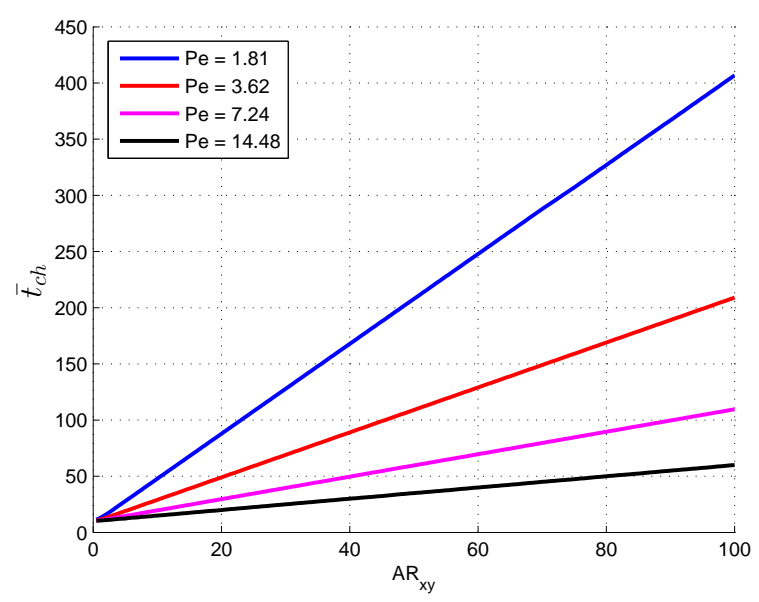

(a) Dimensionless charge time.

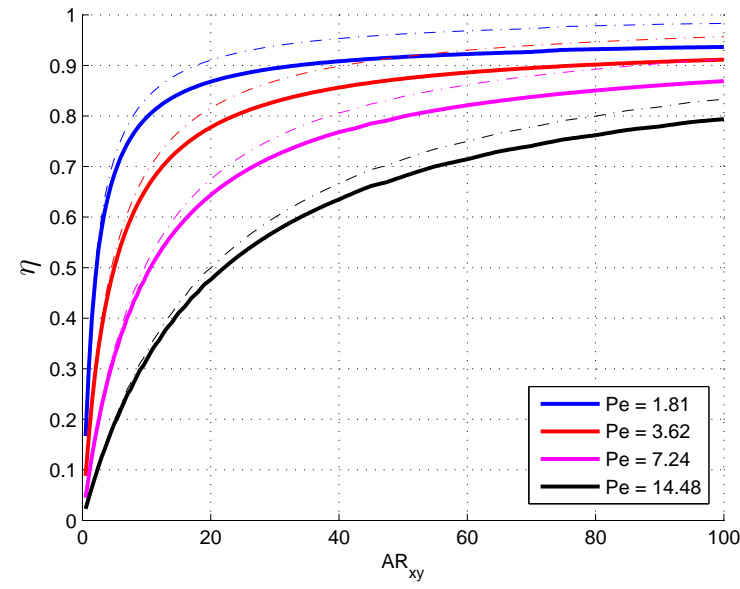

(b) Charging efficiency based on the latent heat stored (solid line) and combined latent and sensible heat stored (dashed line).

Fig. 2 Dimensionless charge time $\bar{t}_{c h}$ and charging efficiency $\eta$ as a function of the PCM-domain aspect ratio $\mathrm{AR}_{x y}$ for different Péclet numbers $P e$.

the charging efficiency with respect to finite water channel heights. Lastly, the performance of multiple stage designs is assessed.

Simulations are all performed in two dimensions $\left(\mathrm{AR}_{x z} \rightarrow 0\right)$ with the domain initialized on the melting temperature $(\bar{T}=0)$ and a fixed Stefan number $($ Ste $=0.05)$

\subsection{Limit case: $\psi \rightarrow 0$}

Consider a water channel with height $H$ being negligible with respect to the PCM domain height $L_{y}$. The total volume of the domain then approximates the PCM volume. The water flow is in very close contact with the PCM and therefore, the heat transfer is not hampered by limited lateral diffusion in the water channel. The dimensional analysis of equation (9) reduces in this limit of $H \rightarrow 0$ to

$$
\bar{t}_{c h}=\frac{\alpha t_{c h}}{L_{y}^{2}}=f\left(\mathrm{AR}_{x y}, 0,0, \text { Ste }, \Pi_{L}, \bar{\rho}_{w}, \bar{k}_{w}, \bar{c}_{w}, 0, P e\right)
$$

In the performed simulations, the supply temperature, PCM properties and water properties are kept constant. Therefore, only two variables are allowed to change: the PCM domain aspect ratio $\mathrm{AR}_{x y}$, which determines the storage shape, and the Péclet number $P e$, which determines the amount of convection and thus the mass flow rate of the hot water. Simulations were performed for different PCM-domain aspect ratios $\mathrm{AR}_{x y}$ and Péclet numbers $P e$. The resulting non-dimensional charge time is shown in figure 2(a).

Apart from the region $\mathrm{AR}_{x y}$ close to zero, the dimensionless charge time shows a clear linear trend with respect to the PCM domain aspect ratio. Two limits of dimensionless charge time are considered. Firstly,

$$
\lim _{\mathrm{AR}_{x y} \rightarrow 0} \bar{t}_{c h}=\bar{t}_{c h, \lim }
$$

with $\bar{t}_{c h \text {,lim }}$ the dimensionless charging time for a 1D PCM domain with dimension $L_{y}$ and a constant wall temperature boundary condition (for the charging limits, see [4]). The wall temperature equals the water input temperature $T_{i n}$. The dimensionless charge time $\bar{t}_{c h \text {, lim }}$ can be determined fully analytically and only depends 
on the Stefan number Ste. Secondly,

$$
\lim _{\operatorname{AR}_{x y} \rightarrow \infty} \bar{t}_{c h}=\bar{t}_{c h, \min } .
$$

The minimum charge time $t_{c h, \text { min }}$ is the least time needed to deliver all heat, which is sufficient to charge the complete storage, which is written as

$$
t_{c h, \text { min }}=\frac{E_{c h}}{\dot{Q}_{i n}}=\frac{\rho_{\mathrm{PCM}}\left(\Delta h+c_{p, \mathrm{PCM}} \Delta T\right) V_{\mathrm{PCM}}}{\rho_{w}\langle v\rangle H c_{w} \Delta T} .
$$

with $\Delta T=T_{i n}-T_{0}$. The minimum charge time in Eq. (14) can be written in dimensionless form as

$$
\bar{t}_{c h, \text { min }}=\frac{\mathrm{AR}_{x y}}{\bar{k}_{w} P e}\left(\frac{1}{S t e}+1\right) \text {. }
$$

Therefore, the trend in figure 2(a) can be drawn for each combination of Stefan number Ste and Péclet number $P e$, apart from the small non-linearity around $\mathrm{AR}_{x y}=0$. Note that dimensionless charge time always goes to infinity for $\mathrm{AR}_{x y}$ going to infinity. Thus, the slope of Eq. (15) is used to determine the trend of figure 2(a). The linear increase of minimum charging time with $A R_{x y}$ can be interpreted as follows: the longer the water channel and PCM domain, the higher the PCM volume, and thus for a fixed input power the higher the minimum charge time. Minimum charge time reduces for higher input powers and therefore also the slope.

The charging efficiency $\eta$ is shown in figure 2(b) and indicates the fraction of heat that has been stored as latent heat. It is clear that higher aspect ratios go along with higher efficiencies, due to the lower diffusion length in the PCM $\left(L_{y}\right)$ and/or longer flow length $\left(L_{x}\right)$ which increases the heat transfer surface. The lower the heat input (lower $\mathrm{Pe}$ ), the higher the efficiency because the output temperature of the water channel reduces. In other words, by slowly charging the PCM storages, the efficiency increases. Note that sensible heat was not included in the definition of the dimensionless charging efficiency. However, temperature gradients exist in the PCM in order to create heat diffusion. By including the sensible heat that is stored in the PCM in the efficiencies definition, the charging efficiency would lie slightly higher. This is shown by the dashed lines in figure 2(b).

\subsection{Influence of $H$ on charging performance}

In reality, it is impossible to produce water channels which are infinitely small, i.e. $H \rightarrow 0$. This would imply that an infinitely large water channel speed is needed for a finite heat input, which is associated with an infinite pressure drop across the channel. Taking these practicalities into consideration, the water channel must have a finite height. The influence of the water volume fraction $\psi$ is therefore investigated in this section. For water channels with a finite height, only the water next to the walls is in direct contact with the PCM. Therefore, lateral temperature gradients are induced in the flow which result in lateral heat diffusion. This means that heat contained in the bulk flow needs to conduct to the wall before it can be stored. Because of this extra heat conduction path, overall charging performance of the storage will be lowered. Simulations were performed for different values of the water volume fraction $\psi$ for a fixed Péclet number Pe. Figure 3 shows the influence of the water fraction $\psi$ on the charging efficiency. As was expected, charging efficiency drops for higher channel widths. Both with respect to power density and storage density, narrow water channels are favoured. The water channel height is therefore limited by production constraints and maximum allowable pressure drop.

\subsection{Multiple stage design}

Previous results all focused on a single stage, i.e. a single water channel and PCM domain. Instead of designing the PCM storage as a single stage, multiple stages could be considered were multiple water channels provide heat to separate PCM domains. The input heat is allowed to be split over all water channels. 


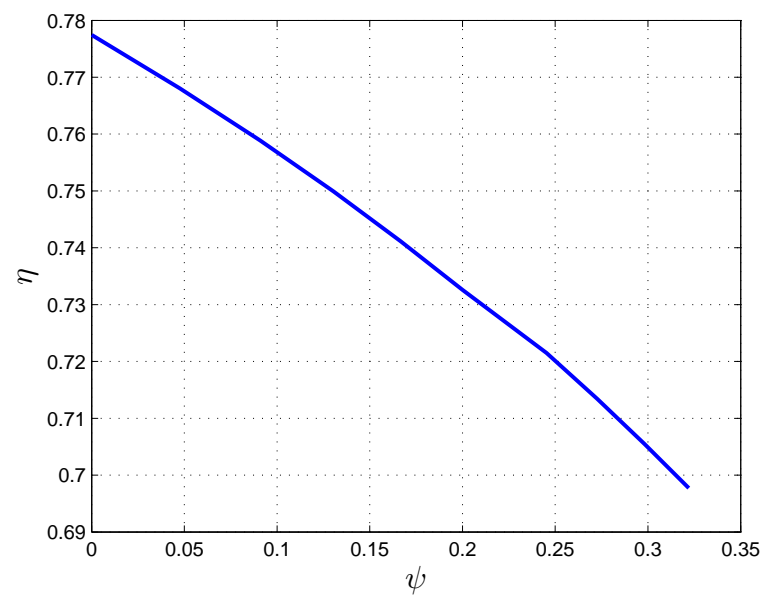

Fig. 3 Charging efficiency in function of the water volume fraction $\psi$ for $P e=3.62$ and $\mathrm{AR}_{x y}=20$.

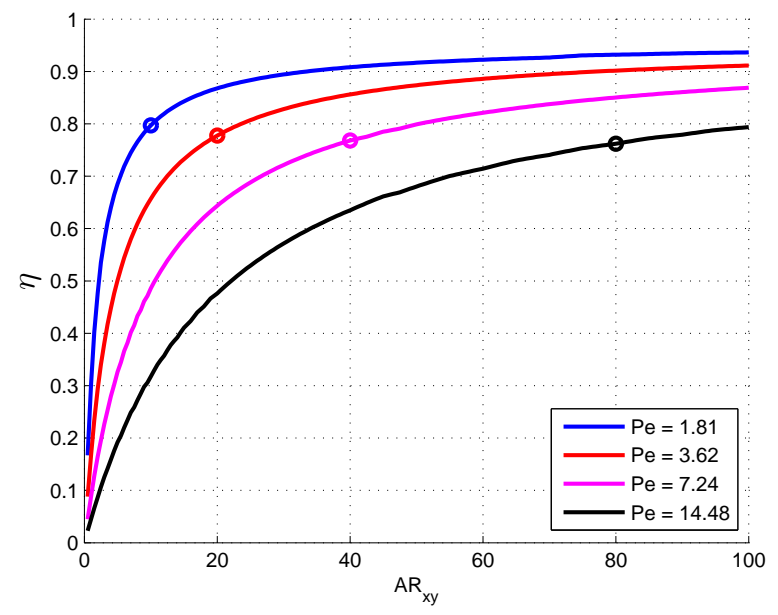

Fig. 4 Comparison of charging efficiency of single and parallelly-charged multiple stage designs.

Figure 1(a) shows the single stage design. By vertically cutting the domain in half and switching the sides in parallel, a multiple stage design is created which still has the same storage density (see figure 1(b)). The individual aspect ratio of the PCM domain of the stages is, however, lower. Charging the stages one after one would result in a lower power density, as was demonstrated in section 5.1. Instead of charging the stages one after one, stages can be charged simultaneously by splitting up the total input power. Dividing the input power uniformly over the water channels, reduces the input power for each channel, and thus also the Péclet number, i.e.

$$
\dot{Q}_{i n, s s}=\frac{\dot{Q}_{i n}}{N} \quad \text { and } \quad P e_{s s}=\frac{\langle v\rangle H}{\alpha_{w} N},
$$

with $\dot{Q}_{i n, s s}, \dot{Q}_{i n}$ the single stage input power and total available power respectively, and $N$ the amount of stages. The charging efficiency (see dots in figure 4) shows a slight increase for higher-stage designs (i.e. lower individual $\mathrm{AR}_{x y}$ and $P e_{s s}$ ). Note that channel pressure drops decrease by an increasing amount of stages.

Multiple stage design enables compact PCM storages to be built with improved charging capabilities. Compactness is achieved by reducing the aspect ratio and increased amount of stages. Stages are either stacked in height (y-direction), in the third direction (z-direction) or a combination of both. Therefore, PCM storages benefit from a high degree of modularity without losses in storage and charging potential. Note that the presented 
multiple stage designs are interpretable as rectangular cuboid PCM encapsulations. Because of the rectangular cuboid shape of the PCM domains, they offer the possibility of being stacked very closely and structured, creating very narrow water channels, and therefore storages with high storage capacities (see Eq. (1)).

\section{CONCLUSIONS}

A parameter study was carried out to study the effect of a finite heat source on the performance of latent heat storages. The heat source was modelled as a water flow with a finite total heat capacity. Both reducing the PCM domain height or increasing the length, improves the charging efficiency. A reduction in charging efficiency was observed for higher Péclet numbers, as the fluid convection is higher. Both with respect to charging performance and storage performance, the channel width should be as small as possible. Production limitations and especially channel pressure drop, which requires pumping power, will restrict the width to some finite dimension. Finally, multiple stage designs were investigated. Aside of creating more compact overall designs, multiple stage designs slightly increase the charging efficiency with respect to a single stage design. Moreover, the splitting of the input power enables the pumping power to be reduced.

The methodology provides relationships for latent heat storage devices, and enables the design of devices with high charging efficiency. For a given storage volume, a multiple stage design can be constructed with high individual PCM domain aspect ratios for increased charging efficiency, provided that the water flow is split uniformly over all stages. Future work includes introducing a separating wall between the PCM and water flow and adding highly conductive fin structures in the PCM, as to design more realistic latent heat storage devices with heat transfer enhancement. To get improved fin lay-outs, numerical optimization strategies will be investigated.

\section{ACKNOWLEDGMENTS}

This research is sponsored by the EFRO-SALK, European Fund for Regional Developments Flanders and Strategic Action plan Limburg Square, Belgium.

\section{REFERENCES}

[1] World Energy Outlook 2014 - Executive summary. International Energy Agency, 2014.

[2] Implementing Agreement on Energy Conversion through Energy Storage Annex 17 - Final report. International Energy Agency, 2005.

[3] B. Zalba, J. M. Marn, L. F. Cabeza, and H. Mehling, "Review on thermal energy storage with phase change: materials, heat transfer analysis and applications," Applied Thermal Engineering, vol. 23, pp. 251-283, 2003.

[4] B. Peremans, M. Blommaert, J. Diriken, and M. Baelmans, "Limits of latent heat storage charging," in Proceedings of the 2nd Thermal and Fluid Engineering Conference, TFEC2017, American Society of Thermal and Fluids Engineers, 2017.

[5] M. D. Muhammad, O. Badr, and H. Yeung, "CFD modeling of the charging and discharging of a shell-and-tube latent heat storage system for high-temperature applications," Numerical Heat Transfer Part A, 2015.

[6] R. Pakrouh, M. Hosseini, A. Ranjbar, and R. Bahrampoury, "A numerical method for PCM-based pin fin heat sinks optimization," Energy Conversion and Management, 2015.

[7] S. L. Lee and R. Y. Tzong, "An enthalpy formulation for phase change problems with a largen themral diffusivity jump across the interface," International Journal of Heat and Mass Transfer, vol. 34, no. 6, pp. 1491-1502, 1991.

[8] P. J. Pritchard, Fox and McDonald's introduction to Fluid Mechanics. John Wiley \& Sons, Inc., 2011.

[9] T. V. Oevelen, Optimal Heat Sink Design for Liquid Cooling of Electronics. PhD thesis, KU Leuven, 2014.

[10] G. Bluman and S. Kumei, Symmetries and Differential Equations. Springer Science + Business Media, LLC, 1989. 